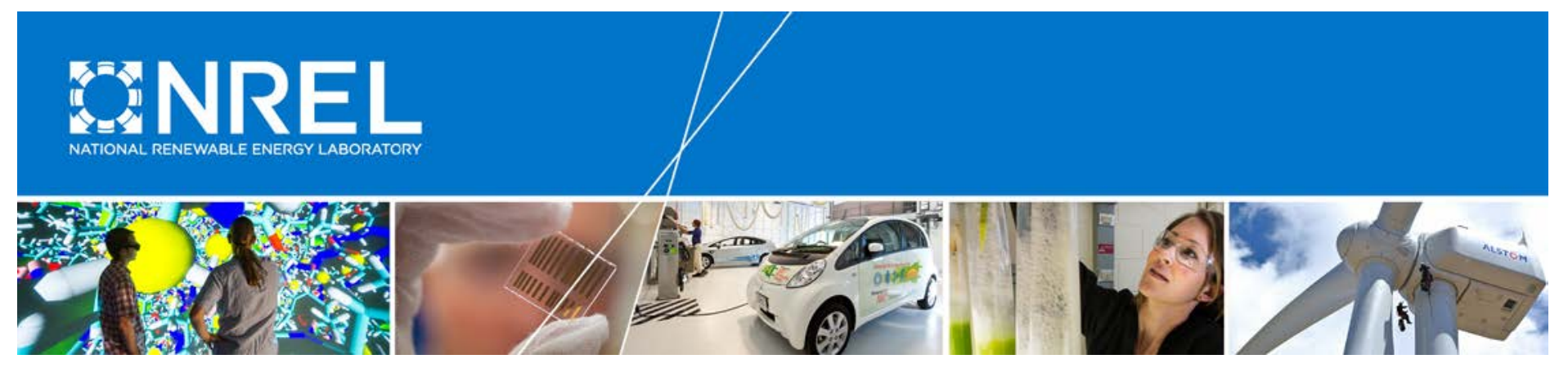

\title{
User-Preference-Driven Model Predictive Control of Residential Building Loads and Battery Storage for Demand Response \\ Preprint
}

Xin Jin, Kyri Baker, Steven Isley, and Dane Christensen National Renewable Energy Laboratory

Presented at the 2017 American Control Conference (ACC) Seattle, Washington

May 24-26, 2017

(C) 2017 IEEE. Personal use of this material is permitted. Permission from IEEE must be obtained for all other uses, in any current or future media, including reprinting/republishing this material for advertising or promotional purposes, creating new collective works, for resale or redistribution to servers or lists, or reuse of any copyrighted component of this work in other works.

NREL is a national laboratory of the U.S. Department of Energy Office of Energy Efficiency \& Renewable Energy Operated by the Alliance for Sustainable Energy, LLC

This report is available at no cost from the National Renewable Energy Laboratory (NREL) at www.nrel.gov/publications.

\section{Conference Paper}

NREL/CP-5500-68037

August 2017 


\section{NOTICE}

The submitted manuscript has been offered by an employee of the Alliance for Sustainable Energy, LLC (Alliance), a contractor of the US Government under Contract No. DE-AC36-08GO28308. Accordingly, the US Government and Alliance retain a nonexclusive royalty-free license to publish or reproduce the published form of this contribution, or allow others to do so, for US Government purposes.

This report was prepared as an account of work sponsored by an agency of the United States government. Neither the United States government nor any agency thereof, nor any of their employees, makes any warranty, express or implied, or assumes any legal liability or responsibility for the accuracy, completeness, or usefulness of any information, apparatus, product, or process disclosed, or represents that its use would not infringe privately owned rights. Reference herein to any specific commercial product, process, or service by trade name, trademark, manufacturer, or otherwise does not necessarily constitute or imply its endorsement, recommendation, or favoring by the United States government or any agency thereof. The views and opinions of authors expressed herein do not necessarily state or reflect those of the United States government or any agency thereof.

This report is available at no cost from the National Renewable Energy Laboratory (NREL) at www.nrel.gov/publications.

Available electronically at SciTech Connect http:/www.osti.gov/scitech

Available for a processing fee to U.S. Department of Energy and its contractors, in paper, from:

U.S. Department of Energy

Office of Scientific and Technical Information

P.O. Box 62

Oak Ridge, TN 37831-0062

OSTI http://www.osti.gov

Phone: 865.576.8401

Fax: 865.576.5728

Email: reports@osti.gov

Available for sale to the public, in paper, from:

U.S. Department of Commerce

National Technical Information Service

5301 Shawnee Road

Alexandria, VA 22312

NTIS http://www.ntis.gov

Phone: 800.553 .6847 or 703.605 .6000

Fax: 703.605.6900

Email: orders@ntis.gov 


\title{
User-Preference-Driven Model Predictive Control of Residential Building Loads and Battery Storage for Demand Response
}

\author{
Xin Jin, Kyri Baker, Steven Isley and Dane Christensen
}

\begin{abstract}
This paper presents a user-preference-driven home energy management system (HEMS) for demand response (DR) with residential building loads and battery storage. The HEMS is based on a multi-objective model predictive control algorithm, where the objectives include energy cost, thermal comfort, and carbon emission. A multi-criterion decision making method originating from social science is used to quickly determine user preferences based on a brief survey and derive the weights of different objectives used in the optimization process. Besides the residential appliances used in the traditional DR programs, a home battery system is integrated into the HEMS to improve the flexibility and reliability of the DR resources. Simulation studies have been performed on field data from a residential building stock data set. Appliance models and usage patterns were learned from the data to predict the DR resource availability. Results indicate the HEMS was able to provide a significant amount of load reduction with less than $20 \%$ prediction error in both heating and cooling cases.
\end{abstract}

\section{INTRODUCTION}

Residential buildings account for $37.6 \%$ of the total electricity consumption in the United States in 2015, higher than all other sectors including commercial buildings, industrial buildings, and transportation [1]. Within the residential building sector, spacing heating, spacing cooling, water heating, and wet cleaning are among the top end-use types and account for about half of the total electricity consumption. These loads are flexible and could be curtailed or shifted at the request of the grid. Residential building loads represent a largely untapped resource for providing grid services such as demand response (DR).

DR programs engage users by reducing or shifting the electricity usage during peak periods to balance the demand and the supply in the electric grid. DR programs include, for example, time-based pricing and direct load control. In direct load control programs, the utility companies or the aggregators cycle home appliances such as air conditioners and water heaters on and off during peak demand periods in exchange for a financial incentive and lower electric bills for the consumer.

Existing DR programs have many limitations. On one hand, utility companies tend to request full control of the

This work was supported by the U.S. Department of Energy Office of Energy Efficiency \& Renewable Energy under contract No. DE-AC3608GO28308 and by the Bonneville Power Administration under Technology Innovation Award TIP 337. The U.S. Government retains and the publisher, by accepting the article for publication, acknowledges that the U.S. Government retains a nonexclusive, paid up, irrevocable, worldwide license to publish or reproduce the published form of this work, or allow others to do so, for U.S. Government purposes.

The authors are with National Renewable Energy Laboratory, Golden, CO 80401, USA. Email: xin.jinenrel.gov appliances to secure their investment and maximize the DR capabilities, which often causes discomfort. Although the grid control may be overridden occasionally, the users could lose financial incentives if they opt out too often. On the other hand, some users are more tolerable to discomfort and their homes are able to provide load reduction more aggressively. The lack of understanding in user preferences hinders the full utilization of the DR potential in residential buildings. Battery storage is becoming an attractive DR tool due to rapidly decreasing battery costs. With a home battery system, the loads could be easily shifted to avoid the peak demand as well as improve the self-consumption of photovoltaic (PV) generation. Due to the higher availability and reliability of the DR resources enabled by a home battery system, utility companies are likely to provide higher incentives to offset the initial capital cost of the battery.

Prior research focused on residential DR using building loads [2], battery storage [3], or both [4], [5]. To the best of our knowledge, user preferences have not been explicitly considered in most of the existing work except [6], where DR scheduling was formulated as a multi-objective mixed integer programming problem. However, appliance models were simplified to combinations of average runtime and energy consumption, and no battery storage was considered. In addition, mixed integer programming problems are computationally intensive to solve and may not be suitable for embedded platforms, such as those needed for cost-effective home energy management system (HEMS) products.

We present a HEMS to address the issues in the existing DR programs and integrate building loads with battery storage to provide more effective DR services with consideration of user preferences. Main contributions of this paper include:

- Introduction of a multi-criterion decision making approach to quickly determine the user preferences on different criteria that are pertinent to user engagement in DR programs;

- Categorization of residential building loads and qualitative analysis of their potential for DR;

- Formulation of residential DR as a multi-objective quadratic programming problem for ease of implementation on embedded or resource-constrained platforms;

- Demonstration of improved flexibility and reliability of residential DR resources with the coordinated control of battery storage and building loads.

The paper is organized into five sections. Section 2 provides an overview of the control architecture as well as the details of the individual components. Section 3 formulates 
the problem as a multi-objective optimization problem and discusses the details of the appliance models. A load shed case study, based on field data from a residential building stock data set, is discussed in Section 4. Section 5 concludes the paper and recommends topics for future research.

\section{System ARChiteCtURE FOR RESIDENTIAL DR}

Residential equipment comprises a variety of devices that have distinct characteristics. Control plays a critical role to maximize the DR potential of the residential resources. In this section, we first define the overall control architecture of the proposed HEMS and then discuss the details of individual components in this architecture.

\section{A. Control Architecture}

A multi-objective optimization problem is formulated to incorporate user preferences of different criteria and coordinate the building loads with battery storage. Figure 1 provides an overview of the system architecture of the proposed HEMS. The workflow for deploying the HEMS in a new home is explained below.

1) Survey: The deployment starts with a brief survey that is generated to elicit the user's preferences over a set of criteria or attributes such as energy cost, thermal comfort, carbon emission, etc. A set of weights of these attributes is derived from the survey and sent to the optimization solver to construct the objective function for the optimization problem.

2) Learning: During the initial period of the deployment, the HEMS collects data about the operating status and power consumption of the appliances and then performs self-learning, which consists of system identification and statistical learning. System identification generates models of the controllable loads for the optimization process. Statistical learning extracts the information that cannot be easily represented as dynamic models, such as the usage patterns of the uncontrollable loads and the water draw patterns.

3) Control: The optimization solver generates optimal solutions for individual devices using the user-preferencedriven objective function, a set of appliance models from system identification, usage patterns from statistical learning, and weather forecast from weather services. Data are collected from the appliances to update the variables in the next optimization step. When a DR signal is received hours before the event starting time, the HEMS generates an estimate of the available DR resources during the DR period and sends the forecast to the utility or aggregator. The HEMS could either pre-cool or pre-heat the building depending on the season and pre-charge the battery to maximize the load reduction. When the HEMS is not providing DR, it could operate in other modes such as energy-efficient mode or lowcarbon mode based on the user's preferences.

\section{B. Residential Appliances}

Many of the end-use types are suitable for residential DR, such as space heating, space cooling, water heating, wet cleaning, etc. These loads are considered controllable loads and make up about $50 \%$ of the total electricity consumption

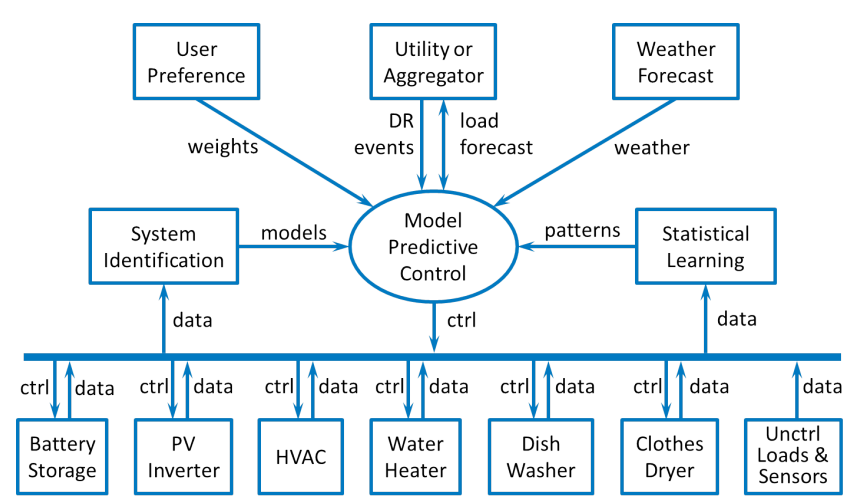

Fig. 1. Control architecture of the HEMS

in the U.S. residential building sector [1]. Other loads such as lighting, refrigeration, cooking, electronics, computers, and miscellaneous loads are either unsuitable or too small for DR. These loads are considered uncontrollable loads since they are not directly controlled by the HEMS.

Among the controllable loads, thermostatically controlled loads such as heating, ventilating, and air conditioning (HVAC) and water heaters are thermal storage devices and can provide large load reduction if pre-cooling or pre-heating is implemented to shift the loads. User-initiated loads such as dishwashers and clothes dryers can be used for DR if delaying the start of these cycles does not adversely affect the user's convenience.

Inverter-controlled power sources such as PV and home battery systems have emerged in the residential building sector. The penetration rate of residential PV has significantly increased over the past few years. Building loads are not always coincidental with the PV generation. With the net metering policy, the excessive PV can backfeed to the grid; otherwise it has to be either curtailed or stored in batteries. Home battery systems can significantly increase the selfconsumption of the PV-generated electricity.

\section{User Preference}

Future HEMS are expected to satisfy users by acting on their behalf through personalized adaptation to their preferences. It is important to understand how the preferences of individuals vary within a probability distribution, what occupants consider ideal, and how they value tradeoffs between the costs and benefits of home services. User preferences vary across the population and change over time. Some occupants value a hot shower above all else, others may be willing to reduce their hot water temperature or shorten a shower occasionally to save money. Acting on behalf of occupants in out-of-sample situations requires a preference-based behavioral model, which is necessary for mass-market acceptance of proposed HEMS. Very little prior research exists on this topic, particularly with regards to multi-criterion decision making problems.

In this paper, we used the Simple Multi-Attribute Rating Technique Exploiting Ranks (SMARTER) method [7] to elicit occupant preferences, where incommensurate multicriterion decisions are required. SMARTER has been shown 


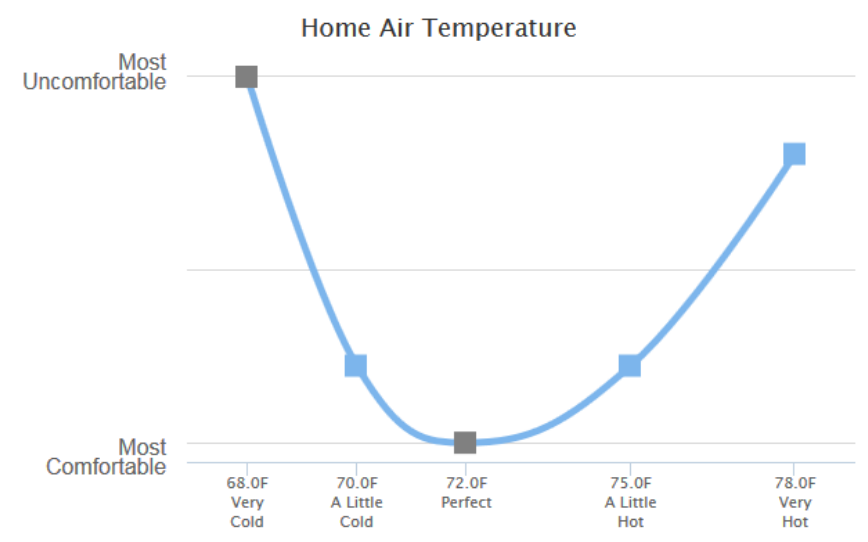

Fig. 2. A temperature profile question from SMARTER survey

to yield superior performance in preference elicitation than other methods such as analytic hierarchy process and discrete choice modeling [8]. SMARTER quickly creates a decision model and avoids other methods' most cognitively difficult task of weighting attributes relative to each other. Rather than ask users enough questions to quantify how much attribute A is preferred to B, users simply rank the attributes, and weights are inferred. For example, users are first asked if being too cold or too hot was worse, then are guided to define their own personal temperature sensitivity curve as shown in Figure 2. Other variables are explored in a similar manner. Finally, the users rank the disparate home services such as home air temperature, shower temperature and length, status of laundry and dishes, as well as financial and environmental costs, to indicate relative preferences. The HEMS then converts the subjective ranking to a set of numerical weighting factors in the objective function.

\section{System Identification}

To accurately predict the behavior of controllable loads with a given control command, it is critical to obtain a model to represent the dynamics of an appliance. The house envelope model and the associated HVAC model are of particular interest not only because space cooling and heating are the largest loads in the residential building sector, but also because each home is highly customized and no default model can accurately predict the HVAC power and indoor temperature without performing model calibration in the actual home. Many system identification techniques have been developed for buildings applications; a review of the techniques can be found in [9].

In this paper an equivalent circuit model of one resistorcapacitor pair is used to represent a single-zone house model and the coefficients can be obtained using multivariate linear regression. We use the learned house model to update the indoor temperature and power consumption based on the outdoor temperature, solar irradiance, and thermostat setpoint. The indoor temperature is measured at or near the thermostat. The outdoor temperature and solar irradiance forecast are available from most weather service companies.

Quadratic programming is used to solve the optimization problem, which means the optimal solutions for the HVAC and water heater are duty cycle commands in fractional form instead of binary variables. The appliances convert the duty cycle commands to on-off control signals using time slot techniques with consideration of minimum on and off time.

User-initiated loads such as the dishwasher and the dryer are also important for predictions in the optimization process. Simplified models are learned from the data to represent the appliances using average runtime and power consumption.

\section{E. Statistical Learning}

Statistical learning is used to learn the patterns in uncontrollable load and hot water draws, both of which are important for the HEMS to make accurate prediction of future load and plan the DR resources accordingly. Statistical methods such as a simple averaging method or more sophisticated machine learning methods such as Expectation Maximization of Gaussian Mixture Models [10] can be used to learn the usage patterns. As an initial attempt, a simple averaging method was used to represent the load patterns at 30-minute intervals. The daily load patterns are represented by 48 bins, and the value of each bin is the average of the training data at the corresponding 30-minute interval.

Uncontrollable loads are small compared to other major loads, but still consume a significant amount of energy. Hot water draws are the driving force of water heater energy consumption and are critical to the accurate prediction of water heating loads.

\section{F. Resource Forecast}

A unique capability of the proposed HEMS is that it is able to provide DR resource forecast for the upcoming DR event. Upon receipt of the DR notification, the HEMS performs look-ahead prediction and computes the loads during the DR period. The prediction is then sent to the utilities or the aggregators, who will be able to make adjustment to the remaining DR calls as well as the generation side. A transactive market can also be created to enable transactions of DR resources among the participating homes.

\section{Problem Formulation}

Management of residential loads and the battery storage is formulated as a multi-objective model predictive control problem. The cost function formed over the considered prediction horizon $H$,

$$
\min _{x} \sum_{h=0}^{H-1} f_{h}(\mathbf{x}(h), \mathbf{U}(h)),
$$

is the sum of the objective function at each time in the horizon $h$, which includes penalties on thermal discomfort due to air and water temperature violations, curtailing PV, and cost of electricity, dictated by the results from the userpreference survey.

The vector $\mathbf{x}$ is a set of time-dependent variables representing the equipment status such as the indoor air temperature, hot water temperature, net load of the home, and available solar power. At each prediction step, $\mathbf{x}$ is updated by the control actions $\mathbf{U}$, which are the optimization 
variables, including the duty cycles of the HVAC and water heater, battery charging and discharging power, and the PV curtailment. The constraints on the optimization problem can be broken down as:

1) Battery Dynamics

2) Single-Zone House Model Heat Balance

3) Water Heater Dynamics

4) Limits on PV Generation and Curtailment

5) Overall Home Energy Balance

During a DR event, to disincentivize using grid power and to incentivize pre-charging the battery or pre-cooling the building before the event, a multiplier is placed to penalize the power drawn from the grid. Figure 1 shows the framework of the optimization problem and the relationship between different modules. Constraints 1) - 5) are explained in greater detail as follows.

\section{A. Battery Dynamics}

The state of charge $B$ and power charged/discharged from storage $P_{b}$ are related as follows:

$$
\begin{aligned}
B(h+1) & =B(h)+\eta_{b}^{c h} \Delta t P_{b}^{c h}(h)+\frac{\Delta t P_{b}^{\text {dis }}(h)}{\eta_{b}^{\text {dis }}} \\
B_{\min } & \leq B(h+1) \leq B_{\max } \\
-P_{b, \max }^{\text {dis }} & \leq P_{b}^{\text {dis }}(h) \leq 0 \\
0 & \leq P_{b}^{\text {ch }}(h) \leq P_{b, \text { max }}^{\text {ch }}
\end{aligned}
$$

where $\eta_{b}^{d i s}$ and $\eta_{b}^{c h}$ are the discharging and charging efficiency of the battery system, $P_{b}^{\text {dis }}$ and $P_{b}^{c h}$ are the discharging and charging power of the battery system, $\Delta t$ is the simulation time step, $B_{\min }$ and $B_{\max }$ are the minimum and maximum state-of-charge (SOC) of the battery in kilowatthours. $P_{b, \text { max }}^{\text {dis }}$ and $P_{b, \text { min }}^{c h}$ are the maximum discharging and charging power.

\section{B. House Model}

The indoor temperature in the house is updated as follows:

$$
\begin{array}{r}
T_{\text {in }}(h+1)=T_{\text {in }}(h)+\beta_{1}\left(T_{\text {out }}(h)-T_{\text {in }}(h)\right) \\
\quad+\beta_{2}\left(I_{H}(h) P_{H}-I_{C}(h) P_{C}\right)+\beta_{3} P_{\text {rad }}(h)
\end{array}
$$

where $T_{\text {out }}$ is the outdoor temperature, $P_{\text {rad }}$ is the solar irradiance, and $\beta_{1}, \beta_{2}$, and $\beta_{3}$ are the coefficients learned from system identification and represent building envelope, heating or cooling gain, and solar gain, respectively. $I_{C} \in$ $[0,1]$ and $I_{H} \in[0,1]$ are the cooling and heating control signals in duty cycle forms. It is assumed that if the HVAC system is in heating mode, then $I_{C}(h)=0$; likewise, if the system is currently in cooling mode, $I_{H}(h)=0$. $P_{H}$ and $P_{C}$ are the delivered heating and cooling power of the HVAC system.

\section{Water Heater Model}

A two-node electric resistance water heater model was chosen for this study based on the authors' previous work [11]. A state space model of the tank dynamics in the water heater is defined as follows:

$$
\begin{aligned}
x(h+1) & =A_{d}(h) x(h)+B_{d}(h) u(h) \\
y(h) & =C_{d} x(h)
\end{aligned}
$$

where $x=\left[T_{W H}^{1}, T_{W H}^{2}\right]^{T}$ is the water temperature at the lower node and upper node of the tank, $u=$ $\left[I_{T 1}, I_{T 2}, T_{i n}, T_{\text {mains }}\right]^{T}$ represents the inputs to the tank including control signals of the lower and upper nodes, indoor temperature and mains water temperature, $y_{d}$ is the outlet water temperature, the state matrix is

$A_{d}=\left[\begin{array}{cc}-\frac{U A_{1}+\dot{m} C_{p}}{C_{1}} & 0 \\ \frac{\dot{m} C_{p}}{C_{1}} & -\frac{U A_{2}+\dot{m} C_{p}}{C_{2}}\end{array}\right]$, the input matrix is
$B_{d}=\left[\begin{array}{cccc}\frac{\eta_{c} P_{W H}}{C_{1}} & 0 & \frac{U A_{1}}{C_{1}} & \frac{\dot{m} C_{p}}{C_{1}} \\ 0 & \frac{\eta_{c} P_{W H}}{C_{2}} & \frac{U A_{2}}{C_{2}} & 0\end{array}\right]$, and the output matrix is $C_{d}=\left[\begin{array}{ll}0 & 1\end{array}\right], U A_{i}$ is the heat loss coefficientarea product, $\dot{m}$ is the flow rate of hot water draws, $C_{p}$ is the heat capacity of water, $C_{i}$ is the thermal capacitance of tank nodes, and $\eta_{c}$ is the efficiency of the resistive element.

\section{PV Constraints}

The PV array was assumed to be $20 \mathrm{~m}^{2}$ with an efficiency of $20 \%$ and tilt of 36 degrees. The curtailment parameter $U_{\text {curt }} \in[0,1]$ denotes the amount of real power curtailed, with 0 denoting no curtailment and 1 denoting a curtailment of $100 \%$. Thus, the PV power consumed by the house can be written as a function of the curtailment parameter and the available solar power, $P_{\text {sol }}$ :

$$
P_{p v}=\left(1-U_{\text {curt }}\right) P_{\text {sol }}
$$

\section{E. Overall Power Balance}

The total load in a home can be denoted as:

$$
\begin{aligned}
P_{\text {load }} & =I_{C} P_{C}+I_{H} P_{H}+I_{T 1} P_{W H}+I_{T 2} P_{W H} \\
& +I_{d w} P_{d w}+I_{c d} P_{c d}+P_{m i s c}
\end{aligned}
$$

where variable $P_{\text {misc }}$ denotes the estimate of uncontrollable, miscellaneous home loads (lighting, television, plug loads, etc.). Clothes dryers and dishwashers are user-initiated, and if initiated during a DR event, are deferred until after the DR event ends. $I_{c d}$ and $I_{d w}$ are binary variables indicating if the cycles are delayed at each prediction time step, and $P_{c d}$ and $P_{d w}$ are the power consumption of these devices.

Then, the overall power balance of the home is as follows:

$$
-P_{\text {grid }}+P_{\text {load }}+P_{b}^{c h}+P_{b}^{\text {dis }}-\left(1-U_{\text {curt }}\right) P_{\text {sol }}=0
$$

for each time step in the horizon (time indices omitted).

\section{F. Objective Function}

The considered objective function seeks to minimize the following at each time step in the horizon:

$$
\begin{array}{r}
f_{h}(\mathbf{x}, \mathbf{U})=b_{t}\left[T_{i n}-T_{a i r}^{\max }\right]_{+}^{2}+b_{t n}\left[T_{a i r}^{\text {min }}-T_{i n}\right]_{+}^{2} \\
+b_{B 1}\left[T_{W H}^{1}-T_{W H}^{\text {max }}\right]_{+}^{2}+b_{T 1}\left[T_{W H}^{\text {min }}-T_{W H}^{1}\right]_{+}^{2} \\
+b_{B 2}\left[T_{W H}^{2}-T_{W H}^{\text {max }}\right]_{+}^{2}+b_{T 2}\left[T_{W H}^{\text {min }}-T_{W H}^{2}\right]_{+}^{2} \\
+c_{e} b_{m} P_{\text {grid }}+b_{c} U_{\text {curt }} P_{\text {sol }}
\end{array}
$$


where $[\cdot]_{+}$indicates $\max (0, \cdot), c_{e}$ is the cost of electricity, $T_{\text {air }}^{\text {max }}$ and $T_{\text {air }}^{\text {min }}$ are the upper and lower limits on the air temperature deadband, and $T_{W H}^{\max }$ and $T_{W H}^{\min }$ are the upper and lower limits of the water temperature deadband. Each weight $b$ is derived from individual user preferences.

The objective function $f_{h}$ is a function of the equipment status variables $\mathbf{x}$ and the decision variables $\mathbf{U}$ where

$$
\begin{aligned}
\mathbf{x} & =\left[T_{i n}, T_{W H}^{1}, T_{W H}^{2}, P_{\text {grid }}, P_{\text {sol }}\right] \\
\mathbf{U} & =\left[P_{b}^{c h}, P_{b}^{\text {dis }}, I_{C}, I_{H}, I_{T 1}, I_{T 2}, U_{\text {curt }}\right]
\end{aligned}
$$

\section{CASE STUDY: LOAD SHED}

A case study was performed on field data to demonstrate the effectiveness of the proposed HEMS for DR. The Residential Building Stock Assessment (RBSA) data set [12] was chosen for this study. RBSA was performed by the Northwest Energy Efficiency Alliance to inform future energy planning efforts and energy efficiency utility programs and rebates. Field surveys were conducted on more than 1,850 sites, including 100 submetered single family homes. In the submetered homes, temperature readings and energy metering were recorded every 15 minutes except for the indoor temperature, which was recorded at 1-hour intervals.

A number of homes across the entire Pacific Northwest region were selected from the submetered data set for the demonstration. The results presented in this section were from a home located in Eugene, Oregon. This home is equipped with a heat pump for spacing heating and cooling, an electric resistive water heater, a clothes dryer, a dishwasher, and other typical appliances.

DR events are likely to occur on cold winter mornings and hot summer afternoons. The coldest days in the RBSA data set were selected to simulate winter morning DR events. Similarly, the hottest days were selected to simulate summer afternoon DR events. These days were selected to test the performance of the HEMS in the extreme cases. Two weeks' worth of data prior to the DR days were used to learn the house model and the usage patterns. Table I shows the results of system identification and statistical learning. The house model was learned from the two-week training data and used to predict the indoor temperature. The $R^{2}$ values of the indoor temperature in both heating and cooling cases were very close to 1 , indicating the accuracy of the learned house model. Statistical learning methods were used to predict uncontrollable loads and hot water draws. Root mean square errors (RMSEs) of the two variables were also pretty small. Note that the RBSA data set does not have water draw data, so water draw profiles from a different data set were used to bridge the gap. The same draw profile was used in the heating and cooling cases, resulting in the same RMSE value in the last row of the table.

A 12-kWh battery pack was modeled with the SOC constrained to $15 \%-85 \%$ to preserve battery lifetime. It was assumed the battery pack has little degradation when operating in this SOC range. More sophisticated battery lifetime models [13] can be incorporated in future research. The power limit of the battery inverter was $5 \mathrm{~kW}$ and its
TABLE I

RESULTS OF SYSTEM IDENTIFICATION AND STATISTICAL LEARNING IN THE RBSA HOME IN EUGENE, OR

\begin{tabular}{l|l|l|l|l}
\hline Methods & Variables & Metrics & Heating & Cooling \\
\hline System ID & Indoor Temp & $R^{2}$ & 0.89 & 0.85 \\
\hline Statistical & Unctrl Loads & RMSE & $0.52 \mathrm{~kW}$ & $0.40 \mathrm{~kW}$ \\
Learning & Water Draw & RMSE & $4.43 \mathrm{gal}$ & $4.43 \mathrm{gal}$ \\
\hline
\end{tabular}

efficiency was $95 \%$. The battery charge/discharge efficiency was $99 \%$.

To solve the quadratic programming problem described in Section III, we used a tool called CVX that is designed for specifying and solving convex programs [14]. The prediction time step was 30 minutes and prediction horizon was set to long enough to cover the entire DR event. The HEMS controls were updated every 30 minutes using the CVX solver, and the appliance simulation was implemented at 1minute intervals.

As mentioned in Section II-F, one of the unique feature of the HEMS is the ability to estimate the DR loads upon receipt of the DR signal. The following metric was used to evaluate the prediction error:

$$
\epsilon=\left|\frac{E_{\text {actual }}-E_{\text {predict }}}{E_{\text {actual }}-E_{\text {baseline }}}\right|
$$

where $E_{\text {actual }}$ and $E_{\text {predicted }}$ are the actual and predicted energy consumed during the DR period when controlled by the HEMS, respectively, and $E_{\text {baseline }}$ is the energy consumed during the DR period when controlled by the traditional hysteresis (or deadband) controller. The same appliance models under the same weather condition were used to simulate the baseline case and generate $E_{\text {baseline }}$.

Figure 3 shows the response of the building equipment controlled by the HEMS to a winter morning DR event. The winter morning load shed DR event, shaded in gray in Figure 3, started at 7:00 a.m. and ended 11:00 a.m. Before the DR event started, the HEMS sent out control signals to pre-heat the building and pre-charge the battery. During the DR period, most loads were off except the heat pump, which turned on several times to maintain the thermal comfort. The battery powered the heat pump and the uncontrollable load to minimize grid power usage. The net load of the home occasionally dropped below zero and pushed power back to the grid. This was because the battery control decision variables $\left(P_{b}^{d i s}\right.$ and $P_{b}^{c h}$ ) were updated once per prediction step, which was 30 minutes in this case. Very little PV was generated due to the season. The baseline load is also shown in the figure in the black dashed line, generated by using the traditional controls without a HEMS or battery storage.

In this case study, a 2-hour advance notification for the DR event was assumed in both heating and cooling cases and the HEMS provided the forecast of the load reduction to the utility/aggregator right after the DR notification was received. As shown in Table II, the DR resource forecast error was $17.67 \%$ in the heating case and $10.60 \%$ in the cooling case, following the metric defined in Equation (15). The HEMS achieved significant load reduction. Compared to the baseline 


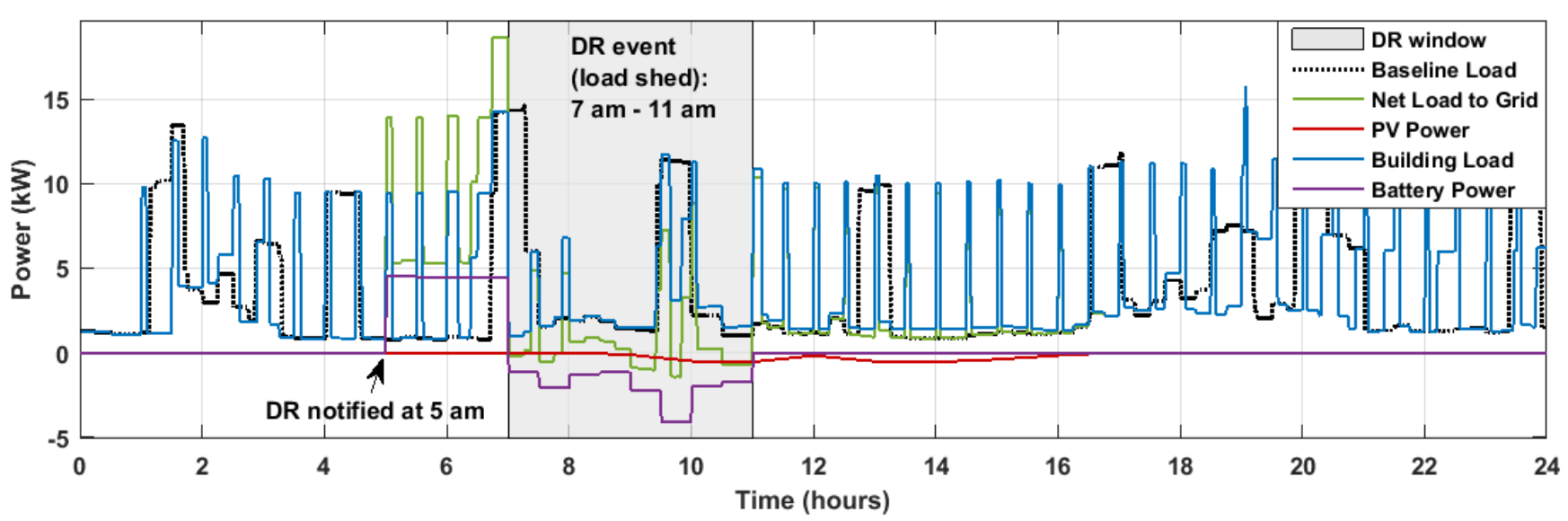

Fig. 3. Power consumption of HEMS-controlled building loads and home battery system responding to a winter morning DR load shed event

TABLE II

SUMMARY OF THE DR RESOURCE FORECAST ERRORS AND ENERGY REDUCTION DURING DR EVENTS IN HEATING AND COOLING CASES

\begin{tabular}{|c|c|c|c|}
\hline & Heating Case & Cooling Case \\
\hline \multicolumn{2}{|c|}{ DR advance notification } & 2 hours & 2 hours \\
\hline \multicolumn{2}{|c|}{ DR start/end time } & $7 \mathrm{am}$ to $11 \mathrm{am}$ & $3 \mathrm{pm}$ to $7 \mathrm{pm}$ \\
\hline \multicolumn{2}{|c|}{ DR forecast error (\%) } & $17.67 \%$ & $10.60 \%$ \\
\hline Energy & Total & $13.13 \mathrm{kWh}$ & $9.45 \mathrm{kWh}$ \\
\hline reduction & Thermal Loads & $5.23 \mathrm{kWh}$ & $4.10 \mathrm{kWh}$ \\
\hline during DR & Battery & $7.90 \mathrm{kWh}$ & $5.35 \mathrm{kWh}$ \\
\hline
\end{tabular}

case, the HEMS provided a reduction of $13.13 \mathrm{kWh}$ in the heating case and $9.45 \mathrm{kWh}$ in the cooling case over the 4hour DR period while still maintaining thermal comfort and meeting other preferences. Both the home battery system and thermal loads contributed to the significant load reduction. In the heating case, the battery capacity was fully utilized by going through full charge and discharge cycles during the winter morning DR event. In the cooling case, the load reduction was smaller and the battery capacity was not fully utilized due to the lower heat pump load when operating in the cooling mode.

\section{CONCLUSIONS}

We presented a solution for user-preference-driven residential DR with building loads and battery storage. A multiobjective optimization problem was formulated and solved by quadratic programming for ease of implementation on embedded platforms. User preference was learned using the SMARTER method and the weights for different attribute terms were incorporated in the objective function. House models and appliance models were obtained using system identification while statistical learning was used to estimate the uncontrollable loads and hot water draw patterns that drove the water heating loads. Simulation results indicate the HEMS was able to accurately predict the DR load reduction a few hours before the event starting time and improve the flexibility and reliability of the DR resources.

Recommended future research topics include:

- Implement the HEMS on a hardware platform and test it with the real appliances;
- Explore other DR use cases such as load-up events when consuming more energy is encouraged by the utility grid;

- Incorporate a battery lifetime model in the objective function to minimize the battery degradation;

- Develop algorithms for optimal sizing of the battery and the PV panel based on a home's historical load data.

\section{REFERENCES}

[1] U.S. Department of Energy, Office of Energy Efficiency and Renewable Energy, "Buildings Energy Data Book," 2011.

[2] I. Hussain, S. Mohsin, A. Basit, Z. A. Khan, U. Qasim, and N. Javaid, "A review on demand response: Pricing, optimization, and appliance scheduling," Procedia Computer Science, vol. 52, pp. 843-850, 2015.

[3] R. Kamyar and M. M. Peet, "A multi-objective approach to optimal battery storage in the presence of demand charges," in International High Performance Buildings Conference at Purdue, West Lafayette, IN, July 11-14 2016.

[4] M. Zheng, C. J. Meinrenken, and K. S. Lackner, "Electricity storage in buildings for residential sector demand response: control algorithms and economic viability evaluation," Columbia University, NY, Tech. Rep. NIST GCR 14-978, June 2014.

[5] R. Batchu and N. M. Pindoriya, Residential Demand Response Algorithms: State-of-the-Art, Key Issues and Challenges. Cham: Springer International Publishing, 2015, pp. 18-32.

[6] R. Jovanovic, A. Bousselham, and I. S. Bayram, "Residential demand response scheduling with consideration of consumer preferences," Applied Sciences, vol. 6, no. 1, p. 16, 2016.

[7] W. Edwards and F. H. Barron, "Smarts and smarter: Improved simple methods for multiattribute utility measurement," Organ. Behav. Hum. Decis. Process., vol. 60, pp. 306-325, 1994.

[8] D. Christensen, S. Isley, X. Jin, K. Baker, P. Aloise-Young, R. Kadavil, and S. Suryanarayanan, "Homeowner preference elicitation: A multimethod comparison: Poster abstract," in ACM BuildSys '16, Stanford, CA, Nov 2016, pp. 243-244.

[9] J. Bloem, Ed., System Identification Applied to Building Performance Data, 1994.

[10] C. Bishop, Pattern Recognition and Machine Learning. SpringerVerlag New York, 2006.

[11] X. Jin, J. Maguire, and D. Christensen, "Model predictive control of heat pump water heaters for energy efficiency," in 2014 ACEEE Summer Study on Energy Efficiency in Buildings. American Council for an Energy-Efficient Economy, 2014, pp. 1-133.

[12] Ecotope Inc., "Residential building stock assessment: Metering study," Northwest Energy Efficiency Alliance, Tech. Rep. E 14-283, 2014.

[13] E. Raszmann, K. Baker, D. Christensen, and Y. Shi, "Modeling stationary lithium-ion batteries for optimization and predictive control," in Proceedings of the 2017 Power and Energy Conference at Illinois (PECI), 2017.

[14] M. Grant and S. Boyd, "CVX: MATLAB software for disciplined convex programming, version 2.1," http://cvxr.com/cvx, Mar. 2014. 